\title{
Interaural correlation and the binaural summation of loudness ${ }^{\text {a) }}$
}

\author{
Barrie A. Edmonds ${ }^{\text {b) }}$ and John F. Culling ${ }^{\text {c) }}$ \\ School of Psychology, Cardiff University, Tower Building, Park Place, Cardiff CF10 3AT, United Kingdom
}

(Received 14 March 2008; revised 16 March 2009; accepted 26 March 2009)

\begin{abstract}
The effect of interaural correlation $(\rho)$ on the loudness for noisebands was measured using a loudness-matching task in naïve listeners. The task involved a sequence of loudness comparisons for which the intensity of one stimulus in a given comparison was varied using a one-up-one-down adaptive rule. The task provided an estimate of the level difference (in decibels) for which two stimulus conditions have equal loudness, giving measures of loudness difference in equivalent decibel units $\left(\mathrm{dB}_{\text {equiv }}\right)$. Concurrent adaptive tracks measured loudness differences between $\rho=1,0$, and -1 and between these binaural stimuli and the monaural case for various noisebands. For all noisebands, monaural stimuli required approximately $6 \mathrm{~dB}$ higher levels than $\rho=1$ for equal loudness. For most noisebands, $\rho=1$ and $\rho=-1$ were almost equal in loudness, with $\rho=-1$ being slightly louder in the majority of measurements, while $\rho=0$ was about $2 \mathrm{~dB}_{\text {equiv }}$ louder than $\rho=1$ or $\rho=-1$. However, noisebands with significant high-frequency energy showed smaller differences: for $3745-4245 \mathrm{~Hz}, \rho=0$ was only about $0.85 \mathrm{~dB}_{\text {equiv }}$ louder than $\rho= \pm 1$, and for $100-5000 \mathrm{~Hz}$ it was non-significantly louder (perhaps $0.7 \mathrm{~dB}_{\text {equiv }}$ ).
\end{abstract}

(C) 2009 Acoustical Society of America. [DOI: 10.1121/1.3120412]

PACS number(s): 43.66.Cb, 43.66.Pn, 43.66.Ba [RYL]

Pages: $3865-3870$

\section{INTRODUCTION}

When a sound is presented binaurally it is perceived to be louder than when it is presented to one ear only (monaurally) at the same sound level. This effect is called binaural summation of loudness (Reynolds and Stevens, 1960). The increase in loudness in the diotic case (i.e., for a stimulus with $\rho=1$ ) is well established for a range of stimuli and sound levels. Early estimates suggested that a sound presented diotically would be heard as being twice as loud (approximately equivalent to a $10-\mathrm{dB}$ increase in sound level) as the same sound presented monaurally (Fletcher and Munson, 1933; Stevens, 1955). More recent estimates (Zwicker and Zwicker, 1991; Sivonen and Ellermeier, 2006; Whilby et al., 2006) have placed the diotic-monaural loudness ratio at somewhat less than 2:1 and equivalent to an increase in sound level of only 3-8 dB. However, data on the effect of presenting a sound dichotically (for instance, with independent noise at each ear) are sparse.

The only previous studies to directly examine the effects of $\rho$ on loudness (Dubrovskii and Chernyak, 1969; Dubrovskii et al., 1972; Eichenlaub et al. 1996) used a loudness-matching paradigm to measure the effect of $\rho$ on the loudness of wide-band noises. The listener adjusted the intensity of one stimulus until it matched the loudness of a second with a different $\rho$. They found that there was no effect of $\rho$ on binaural loudness.

\footnotetext{
Portions of this work were presented at the International Symposium on Hearing, Cloppenburg, Germany, and appeared in Hearing: From Sensory Processing to Perception (Springer, Heidelberg, 2007).

${ }^{b}$ Present address: MRC Institute of Hearing Research, University Park, Nottingham NG7 2RD, United Kingdom.

${ }^{c)}$ Author to whom correspondence should be addressed. Electronic mail: cullingj@cf.ac.uk
}

Notwithstanding this negative outcome, other evidence suggests that an effect might be found. For instance, Zwicker and Zwicker (1991) provided some indirect evidence using magnitude estimation. They showed that a $20 \%$ increase in the loudness estimate of a continuous monaural noise is produced by rapidly alternating the stimulus from one ear to the other. While this is not a direct manipulation of, $\rho$ at high alternation rates, this stimulus will have a $\rho$ of around zero within a finite temporal analysis window, provided that this window is longer than the period of alternation. Zwicker and Zwicker (1991) found their effect at alternation rates of $7 \mathrm{~Hz}$ and over, which is consistent with the $50-200-\mathrm{ms}$ duration of binaural temporal windows reported in the literature (Kollmeier and Gilkey, 1990; Culling and Summerfield, 1998; Holube et al., 1998; Akeroyd and Summerfield, 1999). In addition, Perrott and Buell (1982) showed that interaural correlation can affect the "sound volume" of a noise presented over headphones. Due to the obvious ambiguities in using the word "volume," participants were asked to report the apparent "size" of the sound they heard, and this metric was found to be affected by interaural correlation, by stimulus duration, and by stimulus intensity. The overlapping influence of correlation and intensity upon the same reported metric suggests some degree of perceptual interaction between these stimulus properties.

Moreover, one might also expect $\rho$ to affect loudness from a theoretical standpoint. Several studies have demonstrated that the rate of loudness growth with increasing intensity is greater for tones presented in the NoSo than in the $\operatorname{NoS} \pi$ binaural configuration (Hirsh, 1948; Townsend and Goldstein 1972; Soderquist and Shilling, 1990; Zwicker and Henning, 1991) or in NoSo than in NoSm (Marks, 1987). It is assumed by those authors and in this article that the signal is perceived as a separate entity from the background noise 
and possesses its own partial loudness. Detection of the signal in broadband noise is then simply the starting point of measurable growth in the partial loudness of that signal. Given these assumptions, the differing rate of loudness growth is, in a sense, logically inevitable. At detection threshold NoSo and NoS $\pi$ tones may differ widely in intensity, but be equal in their partial loudness. However, if intensity is then increased for both tones by, say, $20 \mathrm{~dB}$, to a point where the noise becomes relatively insignificant, their difference in intensity leads to a very clear difference in partial loudness (Soderquist and Shilling, 1990). As intensity increases, therefore, a greater rate of increase in loudness occurs for the So tone than for the $\mathrm{S} \pi$ tone. At the same time, a number of authors have pointed out that stimuli in the $\operatorname{NoS} \pi$ binaural configuration have reduced $\rho$ at the signal frequency, which may act as a cue to tone detection (Osman, 1971; Durlach et al., 1986). Combining these two observations leads to the predictions, first, that equivalent manipulation of $\rho$ for a narrow sub-band of an otherwise diotic noise should create the impression of an equally detectable tone embedded within the noise (Jain et al., 1991) and, second, that an inverse relation should exist between $\rho$ in the manipulated band and the partial loudness of the perceived tone (Culling et al., 2001, 2003). Both of these predictions have been fulfilled, although there is some doubt whether $\rho$ is the exact statistic employed by the binaural system in binaural unmasking or whether it merely covaries with the residue from cancellation produced by an equalization-cancellation mechanism (Durlach, 1963; 1972; Van de Par et al., 2001; Culling, 2007). In any case, the relation between $\rho$ and loudness should still hold.

Strictly speaking, this theoretical argument applies only to situations in which the conditions of broadband binaural unmasking are simulated. For instance, where reduction in $\rho$ is band-limited to the frequency region around the tone frequency and a diotic noise fills other frequency regions. However, the signal in $\operatorname{NoS} \pi$ might also be a complex sound, such as speech, present at a number of different frequencies, and so resulting in more widespread reduction in $\rho$. The profile of $\rho$ across frequency may then encode the spectral characteristics of the signal (Culling and Summerfield, 1995; Culling et al., 2001). In the present investigation, we consider what happens to the loudness when $\rho$ is manipulated across the entire stimulus spectrum, as though the signal and masker are identical bands of noise.

The effect of $\rho$ on the binaural loudness of narrow- and wide-band noises was measured using a loudness-matching paradigm. Such an approach serves three purposes. First, the use of a loudness-matching paradigm makes the data obtained much more comparable to studies in the literature on the binaural summation of loudness than the loudness discrimination technique used by Culling et al. (2001, 2003). Second, assessing the loudness of noises at various bandwidths allows for a comparison of the contrasting results of Jain et al. (1991), Culling et al. (2001), and Culling et al. (2003) using narrow sub-bands of noise with those of Dubrovskii et al. (Dubrovskii and Chernyak, 1969; Dubrovskii et al., 1972) using broadband noise. Third, by ensuring that the bandwidths chosen for investigation span above and be-

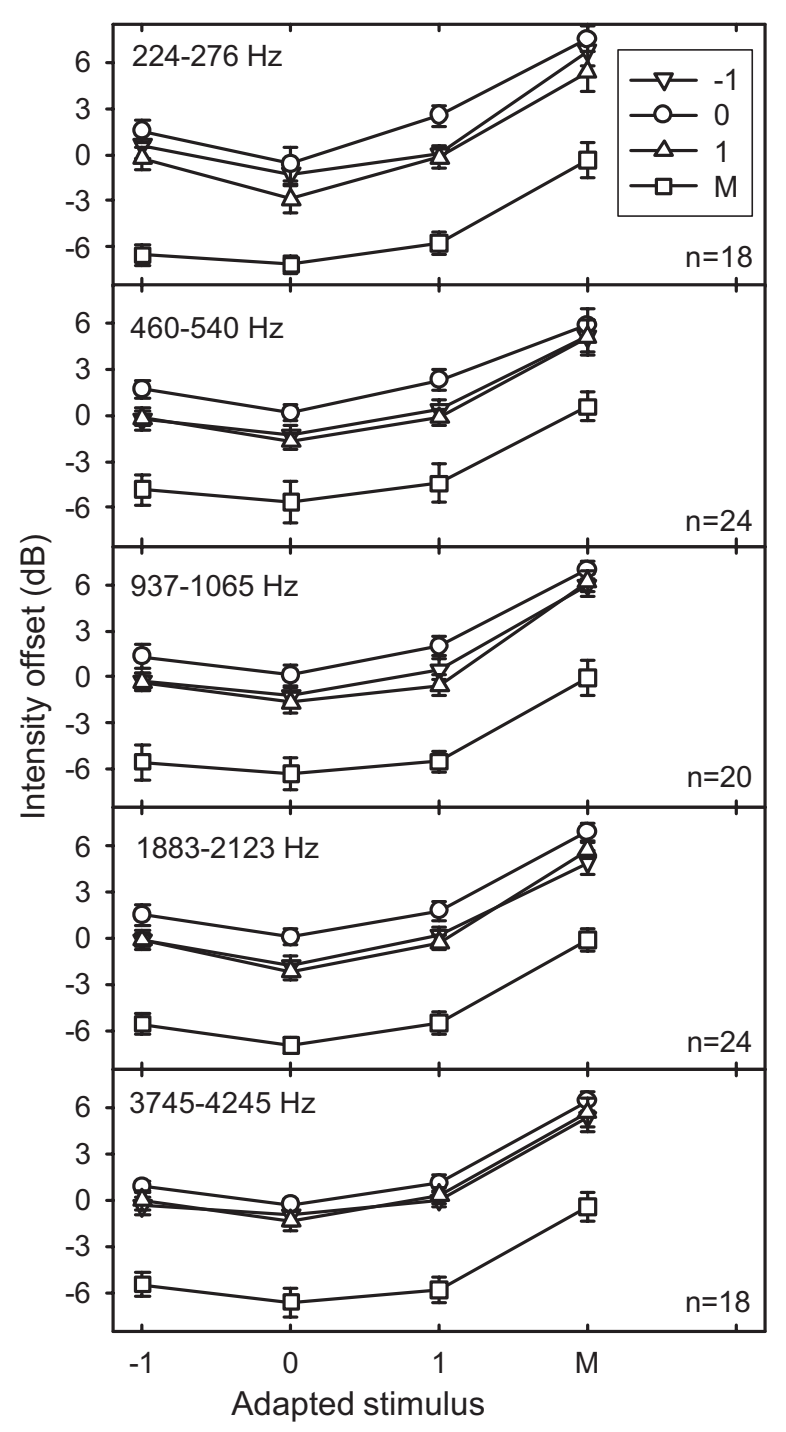

FIG. 1. Intensity offsets needed to match the loudness of noises in different interaural configurations for 1-ERB-wide noise bands at five center frequencies $(250,500,1000,2000$, and $4000 \mathrm{~Hz}$. Binaural configurations are diotic $(1)$, uncorrelated $(0)$, anticorrelated $(-1)$, and monaural $(M)$. Intensity offset is shown for each binaural configuration of reference stimulus as a function of the binaural configuration of the adapted stimulus. Error bars are $95 \%$ confidence intervals. The number of participants contributing data $(n)$ is indicated on the appropriate panel.

low the region important for binaural unmasking one can investigate whether or not the effect of $\rho$ on binaural loudness is linked to the binaural unmasking mechanism.

\section{METHOD}

\section{A. Participants}

Between 14 and 24 paid participants were recruited for each bandwidth condition from the Cardiff University student population using the School of Psychology's participant panel (see Figs. 1 and 2 for exact numbers). Each participant contributed data to only one bandwidth condition. Normal hearing was a specified condition for participation. 


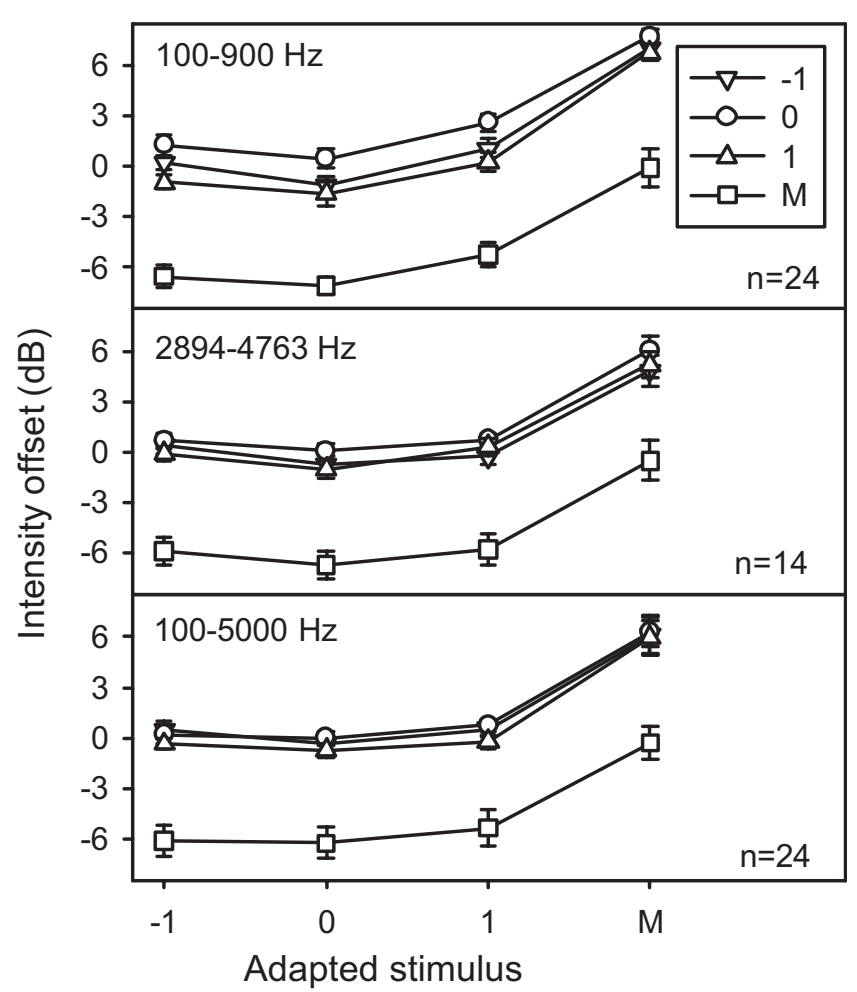

FIG. 2. As Fig. 1, but for the 100-900-, 2894-4763-, and 100-5000-Hz noisebands.

\section{B. Stimuli}

Stimuli were generated online using MATLAB. Broadband noises with a $20-\mathrm{kHz}$ sampling frequency and $500-\mathrm{ms}$ duration were generated digitally and band-pass filtered in the frequency domain by setting the amplitude of all frequencies outside the passband to zero. In order to assess the influence of frequency, five of these bands were 1 ERB wide (Moore and Glasberg, 1983) and centered at 250, 500, 1000, 2000, and $4000 \mathrm{~Hz}$ (i.e., 224-276, 460-540, 937-1065, 1883-2123, and 3745-4245). Three other, wider bands were also investigated. These were a low-frequency band of 100-900 Hz (11.5 ERBs wide), a high-frequency band of $2894-4763 \mathrm{~Hz}$ (4 ERBs wide, centered at $26 \mathrm{ERBs}$ ), and a still wider band of $100-5000 \mathrm{~Hz}$ (25.4 ERBs wide), encompassing both of these regions. These band-pass filtered noises were presented to listeners in four interaural configurations. Monaural $(M)$ noise consisted of a single noise presented to either the left or right ear. Interaurally correlated $(\rho=1)$ noise was generated by presenting the same noise to both ears. Anti-correlated $(\rho=-1)$ noise was created by presenting a noise to the left ear and a phase-inverted copy of that noise to the right ear. Finally, uncorrelated $(\rho=0)$ noise was generated by presenting independent noises at each ear.

The stimuli were presented over Sennheiser HD 590 headphones in a single-walled IAC sound-attenuating booth within a sound-treated room. Digital-to-analog conversion was performed by an Edirol UA20 soundcard and amplified by an MTR HPA-2 headphone amplifier. The intensity of reference stimulus was kept constant at 70-dB SPL, and the adapted stimulus was varied relative to that level.

\section{Procedure}

A two-interval, two-alternative, forced choice adaptive matching paradigm was used to measure the difference in intensity needed to equate in loudness a reference stimulus $(M, 1,-1$, or 0$)$ with an adapted stimulus $(M, 1,-1$, or 0$)$. The order of presentation of the reference and the adapted stimulus was randomly determined on each trial (the listener was unaware of the order of presentation). The intensity of the adapted stimulus was adjusted in accordance with a oneup/one-down rule (Levitt, 1971), producing a staircase pattern of intensity values over the course of the experiment.

Participants were asked to judge which interval contained the louder stimulus. If the participant judged the interval containing the adapted stimulus to be the louder interval, then the intensity of the adapted stimulus on the next trial of that staircase would be less intense. However, if the participant judged the interval containing the reference to be the louder interval then the intensity of the adapted stimulus was increased on the next trial of that staircase. The amount by which the adapted stimulus was adjusted (up or down) was dependent on the number of reversals made while traversing the staircase. The adjustment was $\pm 2 \mathrm{~dB}$ for the first two reversals and then $\pm 1 \mathrm{~dB}$ on subsequent reversals. Each individual staircase was considered complete after 12 reversals using the smaller step size ${ }^{1}$ and was limited overall to 50 trials. This limit was included to limit the overall duration of the experiment if one adaptive track was slow to complete. Subsequent trials were therefore populated by the remaining staircases. A participant's loudness-matched-intensity offset in each of the 16 conditions was taken to be the mean intensity (in decibels) of the adapted stimulus over the last four reversals in each of the corresponding staircases.

Half of the listeners started all staircases with the adapted stimulus $3 \mathrm{~dB}$ more intense than the reference stimulus and the other half started each staircase with the adapted stimulus $3 \mathrm{~dB}$ less intense than the reference. Sixteen adaptive staircases were run concurrently in order to obtain 16 loudness matches (i.e., all pair-wise comparisons of the four interaural configurations with each serving as both reference and adapted stimulus). Trials for different staircases were interleaved at random.

\section{RESULTS}

\section{A. 1-ERB bands}

Mean intensity offsets at loudness match for each of the five center frequencies $(250-4000 \mathrm{~Hz})$ are shown in separate panels in Fig. 1. Each panel shows offsets for the four different interaural configurations of reference stimulus for each interaural configuration of adapted stimulus. An offset of zero indicates that the two interaural configurations did not differ in loudness.

These data were analyzed with separate two-way (reference $\times$ adapted) within-subjects analyses of variance. Each of the five analyses of variance showed rather similar patterns of results. Analyses for each bandwidth indicated significant main effects of both reference and adapted stimulus types $(p<0.0001$, in each case). Both of these results indicate that there is an effect of interaural configuration. 
There was an interaction only for the lowest frequency $[F(9,153), p<0.02]$, which may be neglected after correction of $\alpha$ for multiple tests. It thus made no difference to the results which of the interaural configurations was the adapted stimulus. Tukey HSD post-hoc analyses showed, for both the reference and adapted stimuli, that all levels differed significantly $(p<0.001)$ except for $\rho=1$ vs $\rho=-1$, which was only significant $(p<0.01)$ for the $224-276-\mathrm{Hz}$ band. These differences reflected an effect of binaural summation ( $M$ intensity must be increased to match the loudness of $\rho=1$ ) averaging $^{2} 5.6 \mathrm{~dB}$, and an effect of $\rho(\rho=1$ and $\rho=-1$ intensities must be increased to match the loudness of $\rho=0$ ) averaging $^{2} 2 \mathrm{~dB}$. It is noteworthy, however, that the latter effect reduced to $1.3 \mathrm{~dB}$ for the $4000-\mathrm{Hz}$ center frequency.

\section{B. Wider bands}

Mean intensity offsets at loudness match for each of the three wider bandwidths are shown in Fig. 2 in the same format as in Fig. 1. Each panel shows data for bandwidths of 100-900, 2894-4763, and $100-5000 \mathrm{~Hz}$, respectively. These data were also analyzed with separate two-way analyses of variance. Once again, each analysis produced significant main effects of both the reference and the adapted stimulus $(p<0.0001$, in each case). However, the pattern of results was now somewhat different across the three cases.

Tukey post-hoc analyses indicated that for the low band $(100-900 \mathrm{~Hz})$ and for the high band $(2894-4763 \mathrm{~Hz})$, the pattern was similar to the 1-ERB bands; after correction for multiple tests, each level differed significantly from every other except for $\rho=1$ vs $\rho=-1$. Thus, monaural stimuli must be more intense to match the loudness of $\rho=1$ and $\rho=-1$, while $\rho=0$ must be less intense to match them. The only exception was for the $2894-4763-\mathrm{Hz}$ band, where the -1 and 0 levels were significant only at $\alpha=0.05$. Although this outcome may spring from reduced power in this condition (only 14 listeners were used), the size of the effect was also reduced to $0.9 \mathrm{~dB}$. For the broadest band $(100-5000 \mathrm{~Hz})$, on the other hand, the only levels to differ significantly were monaural vs binaural contrasts $(p<0.001$, in each case).

\section{DISCUSSION}

\section{A. Implications for models of loudness}

Across all of the results, there is a clear effect of $\rho$ on loudness. Current loudness models take no account of $\rho$ (e.g., Zwicker and Scharf, 1965; Moore et al., 1999). Indeed, the current ANSI standard for loudness (ANSI, 2007) is essentially monaural, providing binaural loudness through a simple sum of the monaural responses. However, even in the diotic case the summation of loudness for broadband sounds across the ears is less than perfect and is level-dependent; Reynolds and Stevens (1960) reported that a binaural-tomonaural loudness ratio of 2 (i.e., a doubling of loudness) only occurs at relatively high intensity levels ( $\sim 90$-dB SPL). A recently revised loudness model (Moore and Glasberg, 2007) provides better predictions for the loudness of diotic and dichotically presented sounds, where interaural intensity or frequency differences may exist between the two ears. These predictions are not further affected by changes in $\rho$ of a stimulus. The evidence presented here suggests that models of loudness need modification in order to account for binaural loudness, ${ }^{3}$ particularly in order to model the effect of $\rho$ on loudness at low-frequencies.

Although the effect of $\rho$ may be regarded as small for broadband sounds, it seems likely that this reflects a diluting effect produced by a predominance of high-frequency sound in broadband white noise (see below). In a wide variety of contexts, environmental sound tends to have a predominance of energy at low-frequencies (e.g. Busch-Vishniac et al., 2005; Tang, 1997), making the 2-dB effect more likely to be applicable in practice. Moreover, there are many everyday situations in which one might expect $\rho$ to be low. This may particularly occur in reverberant rooms and in complex listening environments with multiple sounds sources. The effect of $\rho$ may thus be important to consider when assessing levels of noise pollution and in adjusting hearing-aid gain for such situations.

\section{B. Relation to binaural unmasking}

Consistent with expectations based on theories of binaural unmasking, $\rho=0$ was found to be louder than $\rho=1$. This difference is expected on the basis that $\rho$ is reduced when a tone is added in $\operatorname{NoS} \pi$, and as the tone increases in level, the resulting increase in loudness may be encoded via a further reduction in $\rho$. Reduction in $\rho$ across the entire stimulus spectrum would occur if the signal was a sound of equal bandwidth to the masker.

It was also found that $\rho=0$ was louder than $\rho=-1$. Indeed, $\rho=1$ and $\rho=-1$ were found to produce approximately equal loudness. This result can also be accommodated by binaural unmasking theory. If one assumes that $\rho$ is detected after the application of a compensating internal interaural delay within each frequency channel (Durlach, 1963, 1972, Colburn, 1973, 1977; Culling and Summerfield, 1995), then an external correlation of -1 results in an internal correlation, after the compensating delay, that is close to 1 . As the level of the tone in $\mathrm{N} \pi \mathrm{So}$ is increased, this high internal correlation is again reduced. If the experiment is modeled using a $500-\mathrm{Hz}$ gamma-tone filter from the filterbank of Patterson et al. $(1987,1988)$, an internal correlation of 0.98 is predicted for the -1 case. These predictions led Culling et al. (2003) to successfully predict that a $\rho$ of 1 and -1 should be difficult to distinguish in circumstances where their obvious differences in laterality are obscured by flanking bands of noise. For the present data, it is noteworthy that $\rho=-1$ displayed a non-significant trend toward being slightly louder than $\rho=1$, consistent with the slightly lower internal correlation.

Binaural unmasking theory thus provides a viable framework for interpreting the effect of $\rho$ on loudness. However, there are some details of the data which are difficult to fully reconcile with binaural unmasking theory, and binaural unmasking provides only one framework for interpreting this pattern of results. Other possibilities exist (see below).

The difference in loudness between $\rho=0$ and $\rho=1$ is consistently equivalent to only around $2 \mathrm{~dB}$ in intensity. This difference is small in relation to other measurements of sen- 
sitivity to $\rho$. In particular, Culling et al. (2001) found a cumulative $d^{\prime}$ of around 6 for sensitivity to changes in $\rho$ within a 1.3-ERB sub-band of otherwise-correlated broadband noise. While the two measures are not equivalent, the fact that jnds for intensity discrimination tends to be not much below $1 \mathrm{~dB}$ suggests that the $d^{\prime}$ for detecting a 2-dB change would be only 2 or, at most, 3 . A possible explanation of this discrepancy is that listeners are much more sensitive to changes in the spectral profile of $\rho$, as measured by Culling et al. (2001) than to changes in overall $\rho$, as measured in the current study. This interpretation would suggest that discrimination of $\rho$ mirrors the difference between absolute intensity discrimination and profile analysis, where listeners can display a smaller Weber fraction (Green and Mason, 1985).

The results with narrow (1-ERB) bands of noise indicate that the effect is largely independent of frequency. This result is broadly consistent with findings from narrowband binaural unmasking experiments, which show that a large BMLD $(\approx 25 \mathrm{~dB})$ can occur over a wide range of frequencies (McFadden and Pasanen, 1978), although only some listeners appear able to achieve this performance (Koehnke et al., 1986; Bernstein et al., 1998). However, the salient perceptual cue that listeners report in a narrowband BMLD task is intracranial image width rather than the loudness percept which was investigated here, complicating any direct comparison of these findings. Conceivably, increased loudness is not so directly related to binaural unmasking as suggested above. Loudness might occur as a side effect of changes in perceived width. Such an explanation would still be fairly consistent with the pattern of data across different values of $\rho$ because a $\rho$ of -1 , while certainly being perceived as wider than a $\rho$ of 1 , is usually reported as having much less image width than a $\rho$ of 0 (Blauert and Lindemann, 1986); the rank ordering of the conditions, at least, is consistent. This interpretation may be supported by Perrott and Buell's (1982) data on perceived volume of broadband noise. They asked listeners to rate the size of broadband noises presented over headphones and found that listeners' ratings were similarly ordered with respect to correlation as in the current study ( $\rho=0$ being the largest), while at the same time being positively related to stimulus intensity. Correlation and intensity thus contributed to a common construct which must at least be correlated with "loudness." The direction of influence between the constructs of image width, size, and loudness cannot be directly inferred from current data.

The effect of $\rho$ on broader bands shows a marked $(2.1 \mathrm{~dB})$ difference in loudness between 1 and 0 for the lowfrequency $(100-900 \mathrm{~Hz})$ band. This effect is much reduced $(0.74 \mathrm{~dB})$ for the broadband $(100-5000 \mathrm{~Hz})$ case. The simplest explanation for this outcome, and one consistent with the binaural unmasking theory, is that unmasking processes are much reduced for broadband stimuli at frequencies higher than about $1500 \mathrm{~Hz}$, consistent with interaural processing being limited to the use of envelope cues (Bernstein and Trahiotis, 1992). The reduced binaural unmasking of the $1500-5000-\mathrm{Hz}$ region may have diluted the overall effect of $\rho$ on the broadband noise. Indeed, if the effective level in the region up to $1500 \mathrm{~Hz}$ increases by $2.1 \mathrm{~dB}$, and no increase occurs elsewhere, the overall effective level increase is $0.68 \mathrm{~dB}$, very close to the observed figure of 0.74 . Thus, if the high-frequencies show little effect of $\rho$ on loudness, binaural unmasking theory can account for the negative results of Dubrovskii and Chernyak (1969) and Dubrovskii et al. (1972) since their stimuli extended up to at least $5000 \mathrm{~Hz}^{4}$ The only problem for this interpretation is that the 4-ERBwide, high-frequency band $(2894-4763 \mathrm{~Hz})$ displayed an increase in loudness equivalent to $0.86 \mathrm{~dB}$. The effect is somewhat larger than the $0.74-\mathrm{dB}$ effect observed for the $100-5000 \mathrm{~Hz}$, which is inconsistent with an account based purely on dilution.

\section{SUMMARY}

These experiments provide the first direct evidence that interaural correlation can have an effect on the loudness of a binaural stimulus, which can be offset by a compensating difference in physical intensity. The difference in loudness produced by interaural correlation is most apparent at frequencies in the binaural dominance region; the intensity offset required to match in loudness noises that have different interaural correlations at these frequencies is as follows:

(1) Binaural-monaural loudness matches produced mean matched-intensity offsets of about $6 \mathrm{~dB}$, which were consistent with the literature on binaural summation for moderate sound levels.

(2) Noises with an interaural correlation of 0 are louder than noises with interaural correlations of 1 or -1 , the difference being equivalent to about $2 \mathrm{~dB}$ in signal energy in cases for which low-frequencies dominate.

(3) Noises with an interaural correlation of 1 and -1 are closely matched in loudness. Listeners judged anticorrelated noise to be only slightly (and nonsignificantly) louder than correlated noise.

\section{ACKNOWLEDGMENTS}

We would like to thank the reviewers for their help and advice in refining this article. Pilot data for this project were collected by Sonya Ginty. This work was supported by UK EPSRC.

\footnotetext{
${ }^{1}$ In the $500-\mathrm{Hz}$ narrowband condition, only ten trials were used, but this was subsequently extended to 12 for all other data collected.

${ }^{2}$ Averages are across listeners, frequency bands, and the two measurements made with each of the interaural configurations (e.g., 0 and $M$ ) as the adapted stimulus.

${ }^{3}$ There are also data indicating that interaural time delay has some influence on binaural summation (Algom et al., 1988).

${ }^{4}$ Dubrovskii et al. (1972) employed broadband noise limited only by the frequency response of the Russian TD6 audiological headphones they used. They assumed that the response of these headphones rolled off at about $5 \mathrm{kHz}$. However, measurements by Robinson (1971) show that those headphones have a substantial response up to at least $8 \mathrm{kHz}$ with a peak in response at around $6 \mathrm{kHz}$.
}

Akeroyd, M. A., and Summerfield, A. Q. (1999). "A binaural analog of gap detection,” J. Acoust. Soc. Am. 105, 2807-2820.

Algom, D., Adam, R. Cohen-Raz, L. (1988). "Binaural summation and lateralization of transients: A combined analysis," J. Acoust. Soc. Am. 84, 1302-1315.

ANSI (2007). ANSI S3.4-2007. Procedure for the Computation of Loudness 
of Steady Sounds (American National Standards Institute, New York).

Bernstein, L. R., and Trahiotis, C. (1992). "Discrimination of interaural envelope correlation and its relation to binaural unmasking at high frequencies," J. Acoust. Soc. Am. 91, 306-316.

Bernstein, L. R., Trahiotis, C., and Hyde, E. L. (1998). "Inter-individual differences in binaural detection of low-frequency or high-frequency tonal signals masked by narrow-band or broadband noise," J. Acoust. Soc. Am. 103, 2069-2078.

Blauert, J., and Lindemann, W. (1986). "Spatial mapping of intracranial auditory events for various degrees of interaural coherence," J. Acoust. Soc. Am. 79, 806-813.

Busch-Vishniac, I. J., West, J. E., Barnhill, C., Hunter, T., Orellana, D., and Chivukula, R. (2005). "Noise levels in Johns Hopkins Hospital," J. Acoust. Soc. Am. 118, 3629-3645.

Colburn, H. S. (1973). "Theory of binaural interaction based on auditory nerve data I: General strategy and preliminary results on interaural discrimination," J. Acoust. Soc. Am. 54, 1458-1470.

Colburn, H. S. (1977). "Theory of binaural interaction based on auditory nerve data II: Detection of tones in noise," J. Acoust. Soc. Am. 61, 525533.

Culling, J. F. (2007). "Evidence specifically favoring the equalizationcancellation theory of binaural unmasking," J. Acoust. Soc. Am. 122, 2803-2813.

Culling, J. F., Colburn, H. S., and Spurchise, M. (2001). "Interaural correlation sensitivity," J. Acoust. Soc. Am. 110, 1020-1029.

Culling, J. F., and Edmonds, B. A. (2007). "Interaural correlation and loudness," in Hearing: From Sensory Processing to Perception, edited by B. Kollmeier, G. Klump, V. Hohmann, U. Langemann, M. Mauermann, S. Uppenkamp, and J. Verhey (Springer, Heidelberg).

Culling, J. F., Hodder, K. I., and Colburn, H. S. (2003). "Interaural correlation discrimination with spectrally-remote flanking noise: Constraints for models of binaural unmasking," Acta. Acust. Acust. 89, 1049-1058.

Culling, J. F., and Summerfield, Q. (1995). "Perceptual separation of concurrent speech sounds: Absence of across-frequency grouping by common interaural delay," J. Acoust. Soc. Am. 98, 785-797.

Culling, J. F., and Summerfield, Q. (1998). "Mearurement of the binaural temporal window using a detection task," J. Acoust. Soc. Am. 103, 35403553.

Dubrovskii, N. A., and Chernyak, R. I. (1969). "Binaural summation under varying degrees of noise correlation," Sov. Phys. Acoust. 14, 468-473.

Dubrovskii, N. A., Chernyak, R. I., and Shapiro, V. M. (1972). "Binaural summation of differently correlated noises," Sov. Phys. Acoust. 17, 468473.

Durlach, N. I. (1963). "Equalization and cancellation theory of binaural masking-level differences," J. Acoust. Soc. Am. 35, 1206-1218.

Durlach, N. (1972). "Binaural signal detection: Equalization and cancellation theory," in Foundations of Modern Auditory Theory, edited by J. V. Tobias (Academic Press, New York), pp. 369-462.

Durlach, N. I., Gabriel, K. J., Colburn, H. S., and Trahiotis, C. (1986) "Interaural correlation discrimination: II. Relation to binaural unmasking," J. Acoust. Soc. Am. 79, 1548-1557.

Eichenlaub, C., Chouard, N., and Weber, R. (1996). "On the influence of interaural correlation on binaural loudness for broadband noise," in Proceedings of the Internoise '96, pp. 2227-2230.

Fletcher, H., and Munson, W. A. (1933). "Loudness, its definition, measurement and calculation," J. Acoust. Soc. Am. 5, 82-108.

Green, D. M. and Mason, C. R. (1985). "Auditory profile analysis: Frequency, phase, and Weber's Law," J. Acoust. Soc. Am. 77, 1155-1161.

Hirsh, I. J. (1948). "The role of interaural phase in loudness," J. Acoust. Soc. Am. 20, 761-766.

Holube, I., Kinkel, M., and Kollmeier, B. (1998). "Binaural and monaural auditory filter bandwidths and time constants in probe tone detection experiments," J. Acoust. Soc. Am. 104, 2412-2425.

Jain, M., Gallagher, D. T., Koehnke, J., and Colburn, H. S. (1991). "Fringed correlation discrimination and binaural detection," J. Acoust. Soc. Am. 90, $1918-1926$

Koehnke, J., Colburn, H. S., and Durlach, N. I. (1986). "Performance in several binaural-interaction experiments," J. Acoust. Soc. Am. 79, 15581562 .

Kollmeier, B., and Gilkey, R. H. (1990). "Binaural forward and backward masking: Evidence for sluggishness in binaural detection," J. Acoust. Soc. Am. 87, 1709-1719.

Levitt, H. (1971). "Transformed up-down methods in psychoacoustics," J. Acoust. Soc. Am. 49, 467-477.

Marks, L. E. (1987). "Binaural versus monaural loudness: Supersummation of tone partially masked by noise," J. Acoust. Soc. Am. 81, 122-128.

McFadden, D., and Pasanen, E. G. (1978). "Binaural detection at high frequencies with time-delayed waveforms," J. Acoust. Soc. Am. 63, 11201131.

Moore, B. C. J., and Glasberg, B. R. (1983). "suggested formulae for calculating auditory-filter bandwidths and excitation patterns," J. Acoust. Soc. Am. 74, 750-753.

Moore, B. C. J., and Glasberg, B. R. (2007). "Modeling binaural loudness," J. Acoust. Soc. Am. 121, 1604-1612.

Moore, B. C. J., Glasberg, B. R., and Vickers, D. A. (1999). "Further evaluation of a model of loudness perception applied to cochlear hearing loss," J. Acoust. Soc. Am. 106, 898-907.

Osman, E. (1971). "A correlation model of binaural masking level differences," J. Acoust. Soc. Am. 50, 1494-1511.

Patterson, R. D., Nimmo-Smith, I., Holdsworth, J., and Rice, P. (1987). "An efficient auditory filterbank based on the gammatone function," paper presented to the IOC Speech Group on Auditory Modelling at the Royal Signal Research Establishment, 14-15 December.

Patterson, R. D., Nimmo-Smith, I., Holdsworth, J., and Rice, P. (1988). "Spiral vos final report, Part A: The auditory filter bank," Cambridge Electronic Design Contract Report No. APU 2341.

Perrott, D. R., and Buell, T. N. (1982). "Judgements of sounds volume: Effects of signal duration level and interaural characteristics on the perceived extensity of broadband noise," J. Acoust. Soc. Am. 72, 1413-1417.

Reynolds, G. S., and Stevens, S. S. (1960). "Binaural summation of loudness," J. Acoust. Soc. Am. 32, 1337-1344.

Robinson, D. W. (1971). “A review of audiometry,” Phys. Med. Biol. 16, $1-24$.

Sivonen, V. P., and Ellermeier, W. (2006). "Directional loudness in an anechoic sound field, head-related transfer functions, and binaural summation," J. Acoust. Soc. Am. 119, 2965-2980.

Soderquist, D. R., and Shilling, R. D. (1990). "Loudness and the binaural masking level difference," Bull. Psychon. Soc. 28, 553-555.

Stevens, S. S. (1955). "The measurement of loudness," J. Acoust. Soc. Am. 27, 815-829.

Tang, S. K. (1997). "Performance of noise indices in air-conditioned landscaped office," J. Acoust. Soc. Am. 102, 1657-1663.

Townsend, T. H., and Goldstein, D. P. (1972). "Suprathreshold binaural unmasking," J. Acoust. Soc. Am. 51, 621-624.

Van de Par, S., Trahiotis, C., and Bernstein, L. R. (2001). "A consideration of the normalization that is typically included in correlation-based models of binaural detection," J. Acoust. Soc. Am. 109, 830-833.

Whilby, S., Florentine, M., Wagner, E., and Marozeau, J. (2006). "Monaural and binaural loudness of 5-and 200-ms tones in normal and impaired hearing," J. Acoust. Soc. Am. 119, 3931-3939.

Zwicker, E., and Henning, G. B. (1991). "On the effect of interaural phase differences on loudness," Hear. Res. 53, 141-152.

Zwicker, E., and Scharf, B. (1965). "A model of loudness summation," Psychol. Rev. 72, 3-26.

Zwicker, E., and Zwicker, U. T. (1991). "Dependence of binaural loudness summation on interaural level differences, spectral distribution, and temporal resolution," J. Acoust. Soc. Am. 89, 758-764. 